\title{
Georg F. Nicolai: aportes para un estudio de recepción de las ideas de Pavlov en Argentina*
}

Georg F. Nicolai: Aports for Study of Pavlov Ideas in Argentina

Recibido: julio 11 de 2013 | Revisado: enero 17 de 2014 | Aceptado: julio 17 de 2014

\author{
FERNANDO JOSÉ FERRARI ${ }^{* * *}$ \\ Universidad Nacional de Córdoba, Argentina
}

doi:10.11144/Javeriana.upsy13-5.gfna

Para citar este artículo: Ferrari, F. J. (2014). Georg F. Nicolai: aportes para un estudio de recepción de las ideas de Pavlov en Argentina. Universitas Psychologica, 13(5), 1787-1800. http://dx.doi.org/10.11144/ Javeriana.upsy13-5.gfna

Nota de agradecimiento: Agradezco los aportes de Fernando Polanco y Luciano García, quienes generosamente han leído este escrito y han formulado señalamientos que lo han mejorado notablemente.:

** Facultad de Psicología. Docente Jefe de Trabajos Prácticos en la materia, Escuelas Corrientes y sistemas de la psicología Contemporánea. Contacto principal para correspondencia editorial: ferrarijfernando@gmail.com

\section{RES UMEN}

El trabajo se enmarca dentro de los estudios de recepción de las ideas psicológicas en Argentina. Se aborda la figura y la obra del médico y fisiólogo alemán Georg Nicolai, exiliado pacifista que se instala en la ciudad de Córdoba entre 1922 y 1927 . Se estudia el período de formación con el fisiólogo ruso Iván Pavlov y el impacto de la difusión de sus ideas en Córdoba, Argentina. A su vez se estudian las redes intelectuales con las que tuvo contacto y se muestran los espacios institucionales y editoriales que posibilitaron la mencionada difusión.

Palabras clave

Córdoba; Pavlov; recepción; Nicolai

\section{A B S T R A C T}

This is a study about reception of psychological ideas in Argentina. It approaches the figure and work of the German physician and physiologist Georg Nicolai, exiled and settled in the city of Cordoba between 1922 and 1927. We study the training period with the Russian physiologist Ivan Pavlov and the impact of ideas spreading in Cordoba, Argentina. We study the intellectual networks which he had contact with and the institutional spaces that enabled that spreading of psychological knowledge.

Keywords

Córdoba; Pavlov; Reception; Nicolai 


\section{Introducción}

Dentro de la historia de la psicología en América Latina se dieron cita un gran número de psicólogos, científicos e intelectuales que, por diversas razones, migraron desde otras partes del mundo. Este fenómeno trae aparejado la importación de técnicas y saberes que transforman de un modo singular el espacio de producción local. Según Ramón L. (1997), algunos de los representantes de este tipo de difusión fueron los alemanes Walter Blumenfeld (1882-1967, en Perú desde 1935 hasta su muerte), Félix Krueger (1874-1948, en Buenos Aires entre 1906 y 1908) y Carl Jesinghaus (1886-1948, en Buenos Aires entre 1913 y 1935).

En Córdoba, Argentina, también se registró la presencia de científicos e intelectuales europeos. Uno de ellos fue Georg Nicolai; su personalidad y compromiso lo llevaron a exiliarse en Sudamérica en los años veinte. El primer lugar donde Nicolai pudo asentarse fue en la ciudad de Córdoba. Se han realizado pocos estudios de la obra y la vida de Nicolai; cabe mencionar que aunque Zuelzer (1982) ha realizado una biografía, "(...) la biografía de Nicolai describe en forma detallada su vida en Alemania, pero aspectos de sus cuarenta años de vida en Argentina y Chile son descritos con menor detalle y están a la espera de un historiador que los analice" (Cabello, 2013, p. 538). El presente trabajo reconstruye tanto aspectos biográficos como un análisis de las publicaciones que realizara entre 1922 y 1927 , período en que llevó a cabo sus actividades en la ciudad mediterránea, y está orientado metodológica y teóricamente por los principios de una historia crítica de la psicología, propuestos por Kurt Danziger (2010).

El interés se centra en la dinámica de la generación y difusión de los conocimientos psicológicos en el proceso de importación y se aborda una historia de la psicología en términos policéntricos para indagar la migración transcultural de categorías psicológicas. El caso del médico alemán puede encuadrarse en lo que Heilbron, Guilhot y Laurent (2008) han estudiado como mecanismos de difusión transnacional ligados a migraciones intelectuales, más específicamente una migración forzada. Este problema es abordado por los estudios de recepción de las ideas, que pueden ser incluidos dentro del amplio margen que propone la historia intelectual.

En el trabajo de Ruiz, Sánchez y De la Casa (2003), se realiza un análisis crítico de la influencia de Pavlov en América (del norte, fundamentalmente). Para ello, definen tres sentidos en los que puede entenderse tal influencia. 1) el simbólico, al representar un modelo de la posibilidad de construir una psicología objetiva; 2) el metodológico, por la relevancia de la técnica de los reflejos condicionados y 3) el teórico, que se deriva en su teoría de la actividad nerviosa superior. Estos tres sentidos permiten una caracterización de la obra de Nicolai, es decir, delimitar ciertos atributos que distinguen diferentes aspectos de la obra de Pavlov en las producciones de Nicolai.

Para poder abordar la producción escrita de Nicolai, se empleará un criterio provisorio exploratorio, que tiene por objetivo delimitar el modo en que Nicolai adquiere algunos aspectos de la teoría de Pavlov y luego el modo en que los integra a sus escritos, publicados en el período de su estancia en la Ciudad de Córdoba. La obra escrita de Nicolai se dividirá en dos momentos: de 1901 a 1920, la serie de producciones que realiza en Europa y de 1922 a 1927, las producciones realizadas en Córdoba. El primer período está marcado por su formación en medicina fisiológica y un interés en construir una psicología objetiva, es decir, el sentido simbólico de la influencia pavloviana y, fundamentalmente, por aspectos metodológicos. En el segundo período, se puede notar un incremento en los escritos que articulan tanto el sentido simbólico, como el teórico en reflexiones de corte filosófico, en especial una compleja articulación con la teoría de la relatividad de Albert Einstein.

\section{Entre Rusia y Alemania, formación, investigación y política (1901-1920)}

\section{Nicolai, primeras investigaciones con Pavlov}

En este apartado se hará referencia a textos y datos biográficos del período de formación en Alemania y Rusia y los intereses políticos que lo llevan al exilio. 
Nicolai (1874-1964) nace en Berlín un 6 de febrero de 1874. Interesado por las Ciencias Naturales, comienza los estudios de Medicina en Köninsberg, luego continúa su formación en Berlín, París y Heidelberg. Finaliza sus estudios en 1901, en Leipzig, con una especialización como médico fisiólogo. En 1903, comienza a trabajar como profesor asistente en el Instituto Fisiológico de la Universidad de Berlín, en donde aplica el novedoso método de la electrocardiografía, en el que había sido formado por el fisiólogo holandés y futuro premio Nobel, Willem Einthoven. Junto a Friedrich Krauss, introdujo esta metodología en la Clínica médica de la Clínica de la Charité. Ambos fueron partícipes de la primera compilación de un estudio sobre este tema en idioma alemán (Kästner, 2005, p. 201).

En 1906 Nicolai se vio muy afectado por el viaje de estudios que realizó a Rusia para formarse con el fisiólogo Ivan Petrovich Pavlov, quien ya había recibido para ese entonces un premio Nobel por sus investigaciones sobre la fisiología de la digestión. El principal interés de Nicolai siempre fue la aplicación de los resultados de Pavlov para comprender la psicología humana. La admiración por el fisiólogo ruso ya se manifestaba anteriormente, pues Nicolai en 1905 publicó un texto sobre sus investigaciones en la revista Die Medizinische Klinik ${ }^{1}$ [La clínica médica]. Nicolai realizó una estancia de seis meses de formación con Pavlov a partir del 3 de marzo de 1906 en San Petersburgo. En ese período de formación, Nicolai aportó una significativa modificación a las técnicas que el fisiólogo ruso utilizaba para medir la saliva de los perros (Goodwin, 1991); sin embargo, tal como Goodwin resalta, esa modificación se le atribuyó sistemáticamente a Pavlov (véase reproducción de Figura 10.3).

Nicolai deseaba mejorar la forma en que se recolectaba la saliva en los primeros tiempos del laboratorio de Pavlov, cuando las gotas de saliva se contaban sencillamente conforme estas caían en el cilindro graduado que se presentaba en la figura 10.3b. El primer aparato era pesado, problema que reconoció

1 Nicolai, G. (1905): ders., Zu Pawlows. Geburtstag, Die Medizinische Klinik, pp. 259-262.
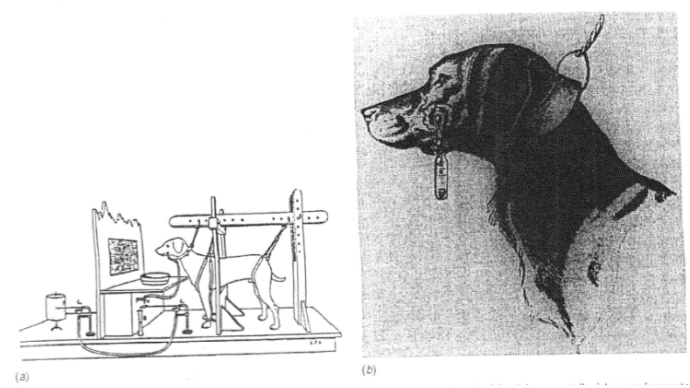

FIGURA 10.3 a) Sistema de condicionamiento desarrollado por el fisiólogo alemán Nicolai, pero atribuido comúnmente a Pavlov. b) Primer dispositivo utilizado por Pavlov para registrar las respuestas salivatorias (ambos tomados de Nicolai, 1907).

Fuente: Goodwin (2009, p. 309)

Pavlov, quien finalmente creó el aparato más sofisticado que se aprecia en la figura 10.3.a. ¿Cómo ocurrió la atribución equivocada del aparato de Nicolai?². (Goodwin, 2009, p. 308)

Al terminar su estadía, volvió a Berlín, en donde comenzó a experimentar con la metodología de Pavlov. Entre tanto, se daría un breve intercambio epistolar con el ruso: los originales de estas cartas se encuentran en San Petersburgo, en la sede del archivo de la Academia Científica Rusa. Kästner (2005) ha logrado reproducir dos cartas en las que se deja ver un profundo respeto por parte de Nicolai

2 "El artículo de Yerkes y Morgulis (1909), en el que se presentaron las investigaciones de Pavlov a los psicólogos estadounidenses, incluía los dos dibujos de la figura 10.3. Ninguno de ellos tenía identificación, pero en el artículo se describía que en la figura $10.3 \mathrm{~b}$ aparecía un perro preparado para las investigaciones sobre condicionamiento en el laboratorio de Pavlov, mientras que en la figura 10.3a se describía "la modificación de la técnica experimental desarrollada por Nicolai en Berlín” (p. 259). John Watson reconoció la diferencia que había entre los procedimientos de Nicolai y de Pavlov, y reprodujo e identificó correctamente ambos dibujos en su conocido texto de psicología comparada de 1914. La primera atribución errónea parece haberse dado en un popular libro de texto introductorio de Walter Hunter (1919); donde solo se incluía la figura 10.3a, la que refirió como el aparato de Pavlov y en la que citó el artículo de Yerkes y Morgulis. Desde entonces, el dibujo del sistema experimental de Nicolai se convirtió en la descripción clásica del aparato de Pavlov, en parte porque es congruente con la separación final que hiciera Pavlov del perro y el experimentador en dos recintos aislados, pero también porque simplemente es un dibujo más interesante que la figura $10.3 \mathrm{~b}$ " (Goodwin, 2009). 
hacia Pavlov. El trabajo de Kästner permite evaluar parcialmente la importancia que Nicolai tuvo en la difusión de las ideas de Pavlov en Alemania y, eventualmente, en Argentina y Chile. En una carta de 1906 se puede leer:

Estimado profesor:

Ayer di un discurso sobre el manuscrito aquí adjunto en el Instituto Psicológico de la Universidad (Psychologischen Institut der Universität). Como usted verá, me he esforzado en explicar el principal valor de su método y luego también, por lo menos en cuanto me fue posible, referir al contenido de la investigación existente. El contenido -que en Alemania es casi desconocido- llamó mucho la atención y desde todos lados se comentó lo importante que sería que este hecho encontrara repercusiones y otros desenlaces. Por ello me pidieron desde mucho lugares que publicara mi conferencia en una revista. Si hiciera esto, lo publicaría en la revista del profesor Vogt, del Instituto neurobiológico (Neurobiologischen Institut) (...). (Nicolai en Kästner, 2005) [S. Kest, Trad.]

Recomendaba a Pavlov que publicase su trabajo en Alemania:

Usted decía aquella vez en Petersburgo que le gustaría (...) publicar sus obras en Alemania, pero que no tendría en vista un periódico y que no sabría cuál podría albergar tantos trabajos suyos. El profesor Nagel me decía que él se sentiría extraordinariamente contento de publicarlo en su "Zeitschrift für Sinnesphysiologie". Yo creo que este lugar no sería inadecuado para la publicación de su trabajo ${ }^{3}$.

Junto con esa carta Nicolai envió a Pavlov su manuscrito titulado: Die Physiologische Methodik zur Erforschung der Tierpsyche, ihre Möglichkeit und ihre Anwendung [El método fisiológico para la investigación de la psiquis animal: su posibilidad y aplicación]. Seguramente, advertido de la particular personalidad del fisiólogo ruso, Nicolai intentó

3 Brief Nicolais an Pavlov vom 1.10.1906, B 1, 1 (links) und Bl.4 (rechts) SPbg. Filial Archiva Ross. Ak. Nauk, f.259, op. 2, N.을 1035, (2-3 ob.) (Kästner, 2005). tomar la precaución de solicitar el aval para su publicación:

Le pido entonces agradecidamente que si quisiera, revise el siguiente manuscrito y eventualmente que me escriba su opinión; si no es mucha molestia, también señáleme los errores presentes. (Nicolai en Kästner, 2005) [S. Kest, Trad.]

Sin embargo, se darían una serie de malos entendidos que terminarían por generar distancia entre ambos médicos fisiólogos. Sin tener ninguna respuesta, Nicolai lo envió a imprimir, tras lo cual fue reprendido con un fuerte rechazo por parte de Pavlov, a quien se le conocía por su enérgico carácter al momento de evaluar los procedimientos y resultados de las investigaciones (Todes, 2003). El prólogo de este texto se publicó en Journal für Psychologie und Neurologie y en Zeitschrift für Hypnotismus ${ }^{4}$. En la segunda carta de Nicolai (quizás de fines de 1907 o principios de 1908, como se puede deducir del contenido) dice lo siguiente:

(...) me decía la señorita Seidemann que usted habría evaluado en tono desfavorable la tendencia de mi trabajo, lo cual me apena terriblemente ya que en realidad en este solamente traté de explayar la increíble importancia que tiene el significado de su método. Es en este mismo sentido que aquí en Alemania se calificó el trabajo antes que nada como un intento para comprobar el valor de su obra para con Kalischer; ya que se concuerda según mi opinión aquí en Alemania, que se sobreestima el trabajo existente de K. Que no haya silenciado mi opinión personal en el artículo (en el cual le adjunto a su método una significación más allá de la que expresa usted mismo y remarco allí que usted podría aprovecharlo con fines psicológicos) es empero autoevidente; y sin embargo le había remarcado expresamente (...) que esto era solo mi visión personal. En lo que resta, importa mucho menos la valoración de los hechos

4 Editado por August Forel, neurólogo y psiquiatra, Oskar Vogt y Korbinian Brodmann, uno de los fundadores de la citoarquitectura del cerebro. 
que los hechos en sí mismos, por lo menos en este sentido. (Nicolai en Kästner, 2005) [S. Kest, Trad.]

Las investigaciones realizadas por Nicolai fueron tomadas en cuenta por Watson en su texto Behavior: An introduction to comparative psychology (1914), editado un año después de su célebre manifiesto Psychology as the behaviorist views it (1913). Allí evaluó diversas investigaciones que Nicolai había realizado en torno a los reflejos condicionados, la luz, los colores y la percepción. De este período no hemos podido relevar todos sus escritos sobre el tema, pero sí algunos que nos permiten tener un primer acercamiento a sus incursiones con la aplicación de la metodología de Pavlov. En el texto introductorio, Watson analiza los estudios realizados con los métodos de Pavlov en relación con el problema de la sensibilidad a las amplitudes de onda (sensitivity to wave-length) y revisa los estudios de Nicolai:

El trabajo más cuidadoso de Nicolai, utilizando el método de Pavlov con filtros de rayos como fuente de luz monocromática, falla al confirmar la perspectiva de que el perro puede responder a diferencias de amplitudes de onda diferencial. El perro, de acuerdo con Nicolai, reacciona en un todo sobre la base de la intensidad. Incluso la evaluación cuidadosa con papeles de colores (Smith) muestran que la sensibilidad del perro a las amplitudes de onda debe ser muy rudimentaria. (Watson, 1914, p. 348) [F. J. Ferrari, Trad.]

La crítica que Pavlov le hacía a Nicolai se debía a un comunicado oral de parte de Vasilij Nicolaevic Boldyrev (Болдырев, Василий Николаевич), quien le habría relatado de manera incorrecta a Nicolai los resultados de la capacidad visual de los perros. Esta problemática la había trabajado Leon Abgaroviê Orbeli (Орбели, Леон Абгарович) en el laboratorio de Pavlov (Kästner, 2005). Posteriormente (Nicolai, 1908), modificaría el error en un trabajo titulado Das Lernen der Tiere (auf Grund von Versuchen mit Pawlowscher Speichelfistel) [El aprendizaje de los animales (con base en experimentos con las glándulas salivales estudiados por Pavlov)].
El trabajo de Nicolai (1908) trata del aprendizaje de los perros a partir de experimentos pavlovianos con fístula salival. Otros trabajos de Nicolai fueron mencionados en sus aspectos metodológicos por Watson (1914, [Introducción]) $)^{5}$. Allí manifestaría el hecho de que los perros no tienen registro de la distinción de colores. Si bien el trabajo de Orbeli no estaba a su alcance en aquellos tiempos, en 1909, aparece en la revista Russische Medicinische Rundschau [Panorama de la medicina rusa], probablemente escrito por Nicolai, una conferencia transcripta sobre la disertación de Orbeli. En las cartas a Pavlov, se encuentran referencias al bochornoso episodio:

(...) en relación a los hechos, se hubiera colado un error (en relación a la capacidad de distinción de colores) me es naturalmente lo más incómodo en todo esto. Yo creía (en base a un comunicado de Boldireff, quien me visitó aquí en Berlín) que los intentos que usted realizaba mientras yo estaba en Petersburgo, recién habían empezado, o que al menos no se habían terminado, y que habrían producido los resultados descriptos por mí. Como yo en este caso (como también en algunos otros puntos) no tenía material impreso sobre el cual respaldarme, me permití en su momento enviarle el manuscrito, pidiéndole que me lo corrigiera; como luego no obtuve correcciones, debí creer que lo expresado en el comunicado estaría retratado tal como usted deseaba. Desde luego voy a apurarme en corregir este error, publicando una nota. Pero por eso quiero dejar en claro que quisiera de manera auténtica-evidentemente he entendido mal a Boldireff- y le estaría sumamente agradecido

5 "Los experimentos de Nicolai, en donde el reflejo salival fue utilizado como indicador, muestra aparentemente, que el perro es sensible a los estímulos mecánicos y que la localización de tales estímulos es precisa" (Watson, 1914). Más aún, dentro del ámbito específico del registro cutáneo de la temperatura, Watson retoma los desarrollos de Nicolai: "Nicolai declara que cuando se aplica frío en un punto en particular de la piel del perro, un reflejo condicionado es provocado. Si el frío es aplicado en cualquier otro punto, el reflejo aparece a continuación, mostrando que la localización no es muy exacta. Por el mismo método se ha mostrado que la sensibilidad del perro a lo cálido y lo frío puede reaccionar de modo diferente a lo cálido" (Watson, 1914, p. 427) [F. J. Ferrari, Trad.] 
si me enviara el trabajo en cuestión. (Nicolai en Kästner, 2005)) [S. Kest, Trad.]

Finalmente se produciría un distanciamiento entre el fisiólogo ruso y Nicolai:

(...) Sobre todo me ha afligido, tomando las palabras de la señorita Seidemann, que el afecto que me hubiera guardado en el pasado hoy ya no estuviera presente. Le pido que sepa que todo aquello que usted pone en reparo acerca de mi trabajo, fue escrito por mi desde las mejores intenciones y que sobre todo en relación al manuscrito pensaba estar seguro de tener su aprobación. Una revisación integral del trabajo tiene que convencerlo de que en éste hay una gran apreciación hacia sus trabajos y si usted se hubiera convencido de esto, hubiera sido amable de su parte que, en reconocimiento de los hechos, me hubiera mandado sus críticas directamente y no por medio de terceros. (Nicolai en Kästner, 2005) [S. Kest, Trad.]

A pesar de su gran afección por las doctrinas de Pavlov, Nicolai bregaba, al contrario que Pavlov, por una psicología que estuviese basada en la nueva metodología de los reflejos condicionados. Aunque es conocido que Pavlov, a pesar de mostrar un cientificismo inflexible, también se dedicaba a realizar especulaciones que trasladaban los resultados de su exclusivo laboratorio a problemas de orden antropológico y hasta filosófico. Pavlov no desconocía que sus teorías eran permeables a tales desplazamientos. Todavía deben ser relevados los textos de Nicolai para evaluar los aportes y limitaciones de sus experiencias con el método de Pavlov. Sin embargo, el haber sido tomadas por Watson indican que significaron avances modestos en torno al uso del reflejo condicionado y su relación con la percepción de la luz y variaciones de temperaturas, así como también al aprendizaje en los animales. Siendo la innovación en la técnica de recolección de la saliva, su aporte de mayor envergadura.

\section{Nicolai pacifista, el exilio}

Al comienzo de la primera guerra mundial, en octubre de 1914, se publica un escrito firmado por 93 intelectuales alemanes llamado Manifiesto al mundo cultural para avalar las acciones bélicas. Nicolai publicó unos días después Manifiesto a los europeos, en el que describía una visión en la que Europa estaría unida en el futuro. Este manifiesto fue realizado junto con Albert Einstein, Otto Buek y Wilhelm Föster. En breve, el doctor Nicolai, comenzó a dar conferencias acerca de las consecuencias de los conflictos bélicos bajo el título: "La guerra como un factor biológico en el desarrollo de la Humanidad", señalando la pérdida de vidas, energía y dinero de la gente. Estas conferencias se fueron recopilando y se convirtieron en el texto The biology of war, cuya escritura concluyó mientras su autor estaba en la prisión de Graudenz. En 1917, se publicó la primera edición del libro en Zürich y, en 1918, se tradujo al inglés. El texto The biology of war (Nicolai, 1918) retomaba los mismos puntos que los ensayos de Einstein sobre las raíces psicológicas de la guerra. En ambos se sostenía que la raza humana debería canalizar sus impulsos agresivos en fines comunitarios (Rowe \& Schulmann, 2007, p. 62).

En el verano de 1915, Nicolai convenció a Einstein para que participara de la New Fatherland Association, una asociación pacifista. En ese espacio, el fisiólogo alemán conoció al pacifista Romain Rolland y a los físicos Henbdrik A. Lorenz y Paul Ehrenfest. La tensión creció y el Gobierno comenzó a perseguir a Nicolai para llevarlo a la corte militar. Albert Einstein le escribe a su amigo Max Born el 27 de enero de 1920: "Nicolai is being attacked and insulted so much that he is no longer able to lecture, not even at the Charité" (Grundmann, 2004, p. 92). Las complicaciones siguieron hasta que, en el verano de 1918, Nicolai voló en un avión militar a Dinamarca. En 1922, fue forzado a dejar Alemania y se exilió en Córdoba, Argentina, y luego en Chile, donde moriría en 1964.

Nicolai en Córdoba, Argentina (1922-1927)

Nicolai arriba a Córdoba en 1922 para trabajar en la cátedra de fisiología experimental de la Facultad de Medicina ${ }^{6}$. Allíse desarrollaron los primeros pasos en

6 La modernización de la cátedra y la fundación del laboratorio de fisiología estuvieron a cargo de Valentín De Grandis, quien había 
psicología y fisiología experimental de la mano del Dr. Virgilio Ducceschi, a partir de 1907 (Ferrari, 2013). Virgilio Ducceschi se mantiene en ese cargo en la Facultad de Medicina de la Universidad Nacional de Córdoba desde 1906 hasta 1918. El 11 de marzo de 1922, se celebró la incorporación de Nicolai y Alfonso Goldsmidth. A partir de allí, desarrollaría su actividad en la cátedra de Fisiología hasta 1927.

\section{Sus producciones escritas}

En Alemania, Wundt había fundado el laboratorio de psicología experimental en Leipzig (1879). La experimentación en psicología no es algo unívoco. Son conocidas las diferencias ente la psicología experimental practicada por Wundt y la que se introduce en Norteamérica. En Alemania, la experimentación en psicología estaba ligada al ejercicio de la introspección profesionalizada y afectada por una perspectiva filosófica. Así mismo, en Francia la experimentación estuvo desarrollada dentro del ámbito clínico y psicopatológico. Como es conocido, esta última línea es la que tuvo mayor impacto en Argentina, de donde Felix Krüeger, discípulo de Wundt, sería prácticamente expulsado por el medio intelectual bonaerense (Klappenbach, 1994). Nicolai no era partidario de las investigaciones de Wundt, pero, del mismo modo en que Krüeger no tuvo lugar en el campo científico argentino, pudo haberse encontrado con un medio poco fecundo en Córdoba que receptara y aplicara sus ideas.

Si bien Nicolai estaba interesado en los procesos sociales y políticos, tomará distancia de la idea de una Völkerpsychologie, que encuentra en Wundt su mayor representante. Wundt es deudor de una línea de pensamiento que Danziger (1983) sitúa en los desarrollos de Lazarus y Steinthal, quienes habían basado parte de sus planteamientos sobre una psicología de los pueblos que Herder había postulado. Wundt no encontraba en la Völkerpsychologie ninguna limitación o inferioridad respecto de la psicología experimental. Más aún, comprendía que era una de las ramas más importantes de la ciencia

\footnotetext{
sido el primer fisiólogo extranjero contratado en Buenos Aires
} para orientar la formación del laboratorio de Horacio Piñero. psicológica y que estaba destinada a eclipsar a la psicología experimental. Ante esto, Nicolai terminaba por criticar no solo a Wundt, sino a Lazarus, Steinthal y a Herbart, señalando una insoportable metafísica? ${ }^{7}$ Su interés por la investigación fisiológica, en cuyos fundamentos encontraba a la física (einsteiniana), lo acercó, como hemos visto, a Pavlov, pero también a una línea de pensamientos en los que, según Nicolai, se prescindía de las aberraciones de la metafísica. Por ello es que es detectable la primacía de las figuras de Spencer, Darwin, Mach, Fechner, Le Dantec. Ese cruce entre fisiología y física lo producirá a partir del problema del tiempo y el espacio, que para Pavlov era fundamental (Windholz, 1995). Nicolai adhería a la idea de Pavlov, al modo en que lo hacían los neokantianos, de que toda psicología debía ser explicada en términos de espacio-tiempo, en donde el sistema nervioso sostenía el espacio mientras que los procesos de condicionamiento eran la medida de tiempo. En ese nexo, como veremos, encontrará el punto de contacto con la Teoría de la Relatividad.

Tres de los textos que produce en Córdoba fueron publicados en la Revista de la Universidad Nacional de Córdoba (RUNC) ${ }^{8}$. Esta inició su publicación en agosto de 1914, bajo el rectorado del Dr. Julio Deheza, dirigida por el Dr. Enrique Martínez Paz. También publicó en la conocida Revista de Filosofía, cuyos espacios servirían para exponer sus aproximaciones a la teoría de la relatividad y sus denuncias a los tratos que recibiera de la cerrada

7 Nicolai se presentaba “(...) contra las tendencias seudo-modernas, que en Alemania representa Wundt, en Francia Bergson, y James en los países de lengua inglesa. Es meritorio fundamentar nueva y exclusivamente la psicología en la biología, que es su única base real, (...). Verdad que tiene los mejores precursores, hombres como Darwin, Spencer, Mach, Fechner, Le Dantec, que han inaugurado este camino" (Nicolai, 1926, p. 88).

8 Fue una revista de periodicidad irregular, sufrió dos interrupciones, la primera de ocho meses, en 1918, por la renuncia del Dr. Enrique Martínez Paz, su director de entonces, reiniciándose con el año V (N.o 4-5, octubre-noviembre de 1918); la segunda se produjo a raíz del incendio que en 1958 destruyó la imprenta de la Universidad. $\mathrm{Al}$ aparecer nuevamente, fue cuando se inició la Segunda Serie, con el año I (N.o 1 marzo-abril de 1960). Mantiene una vigencia de lo sostenido por el Dr. Poviña al definirla como de "tipo enciclopédico. En sus comienzos, tuvo una sección bibliográfica, con notas críticas de libros, crónicas, resoluciones y "revista de revistas", que en su Segunda Serie se conserva para crónicas y críticas bibliográficas. 
estructura conservadora de la Universidad de Córdoba. La RUNC fue el signo de la apertura que la Universidad tuvo en los primeros años de docencia de Nicolai. En ella, se publicaron dos escritos del fisiólogo alemán, el primero titulado Las isopsicas de los animales y la relación entre la inteligencia y el cerebro según observaciones en 192 perros (1923). Nicolai parte de la idea de que hay una relación funcional entre inteligencia y volumen cerebral: "Esta figura y este sistema es la prueba indubitable de que la inteligencia es una función del desarrollo del cerebro en relación con el peso del cuerpo, lo que podríamos expresar matemáticamente" (Nicolai, 1923, p. 36).

Aquí se ponen de manifiesto los principios darwinianos que le permiten pensar a Nicolai la continuidad filo y ontogenética de la vida, desde los organismos menos complejos a los más complejos. Darwin había tenido una profunda influencia en los estudios de Pavlov, en particular a partir de la lectura de su The Expression of the Emotions in Man and Animals (1872). Este Darwinismo destila una influencia insistente en toda la obra de Nicolai. Es desde esa línea que se introducen los problemas de la herencia de caracteres adquiridos, que será uno de los pilares de los planteos de Nicolai. Por otra parte, también encontrará su manifestación en su compromiso con la eugenesia. Tanto Pavlov como Nicolai, a instancias de Darwin, encuentran en la eugenesia una tecnología de purificación del tipo biológico. Todo ello enmarcado por el darwinismo social de los planteamientos de Spencer, le proporcionan a Nicolai las herramientas teóricas para comprender una sociología y una psicologías que buscan ser estrictamente científicas. Intenta establecer una ley general que muestre la correlación entre inteligencia y volumen cerebral. Esto indica que buscaba constatar la idea de que los fenómenos psicológicos, como la inteligencia, dependen de un sustrato fisiológico. Lo cual está relacionado con los aspectos simbólicos de la obra de Pavlov, vale decir, aquellos que inducen a creer en una posible psicología objetiva. Para ello, recurría a trabajos de Parisot y de Spitzka que mostraban esa correlación. "La formación que obtuviese en Rusia con Pavlov, estuvo presente en sus investigaciones: Es evidente que la complejidad de las reacciones psíquicas -que se llaman en sus manifestaciones primitivas reflejo, después instinto y en sus más altos grados inteligencia- sea una función de la complejidad del sistema nervioso central, principalmente del cerebro (Nicolai, 1923, p. 3).

En su segundo texto, intentó fundamentar biológicamente la teoría de la relatividad de Albert Einstein. En este sentido, en 1925, publicó un libro, avalado también por la RUNC titulado La base biológica del relativismo científico y sus complementos absolutos, en donde intenta dar cuenta de las nociones de tiempo, espacio, percepción, sensación e inteligencia, tomando las referencias teóricas de Einstein. Nicolai recurrió a Pavlov, para rebatir los principios kantianos de los a priori de tiempo y espacio. Pavlov mismo había fundado su teoría las actividades nerviosas superiores, a partir de esos $a$ priori, pero aseverando que dependían de la actividad nerviosa (Windholz, 1995).

(...) este libro no pretende ser de física sino de consideraciones fisiológicas. Quiero demostrar que estudiando las bases biológicas de nuestras nociones de tiempo y espacio, masas y fuerzas, se llega a conclusiones que, aunque no tengan la precisión de la fórmula, pueden dar nueva luz a la teoría de la relatividad. (Nicolai, 1925a, p. 14)

Hay límites a nuestra intuición, y ellos son a priori, es decir condicionados por nuestra organización. Naturalmente este a priori no es metafísico sino fisiológico o, si se quiere, físico. (Nicolai, 1925a, p. 30)

Si bien acuerda con Kant en que nada es si no es a través del tiempo y el espacio, argumenta que no son a-prioris, sino que son adquiridos por la experiencia empírica. Esto es resuelto con su compromiso evolucionista. De este modo, tiempo y espacio son heredados de una experiencia vital que proviene de un pasado pretérito que evoluciona hasta el hombre actual. Habiendo explicitado su compromiso con el empirismo, citando a Locke y a Leibniz, argumentará la posibilidad de una nueva "metafísica" de la teoría de la relatividad en la que la ciencia puede, a través de la matematización, superar a las sensaciones. Tiempo y espacio son 
comprendidos en términos filogenéticos, asociados a una teoría de la vida9. Aquí la vida estará asociada al transcurso temporal y la espacialidad que se expresan en una sustancia extensa. De hecho liga la vida psíquica a esa sustancia extensa, lo cual es coherente con su deseo de construir una psicología objetiva y fisiológica.

En el texto hay numerosas referencias a los escritos del español Turró, quien también estudió la doctrina de Pavlov en España (Bandrés \& Llavona, 2003). Tanto Nicolai, como Turró, desarrollan articulaciones filosóficas en torno a las implicancias del método y la teoría de Pavlov para el desarrollo de una psicología científica. Podemos apreciar que esto implica el impacto de las ideas de Pavlov tanto en el sentido simbólico, como en el teórico y se han dejado de lado las implicaciones metodológicas que dominaban el primer período. El diálogo en el que transcurre el texto, incluye una referencia constante a la obra de Kant. Discute además con los planteos filosóficos del vitalismo Ortegiano. En esta articulación filosófica, la psicología es una extensión de la fisiología, y se comprenden las sensaciones como expresiones psicológicas. Luego de referir a sus investigaciones con Pavlov sobre los colores ${ }^{10}$, aclara que esta situación está dada porque los colores son, de las sensaciones, las más recientes y por eso tienen más débiles raíces en nuestra psiquis. Nuevamente, apela a un progreso filogenético que deja de lado a la idea de que existan cosas innatas. Vale decir, sería una ilusión el decir que el tiempo y el espacio son innatos, puesto que fueron adquiridos por la experiencia de generaciones pasadas. De tal manera, "La noción de tiempo es un instinto también" y "Los instintos son reflejos inconscientemente adquiridos

9 En rigor de verdad ya había publicado en Argentina un texto llamado "La vida" en donde desarrollaba planteos sobre el concepto de Vida (Nicolai, 1922). Este texto fue la conferencia del primer día que fue recibido en la Universidad de Córdoba y luego fue publicado en la Revista de Filosofía de Ingenieros. De algún modo, este texto marca un cambio importante, puesto que en él realizaba un análisis filosófico de la vida, que terminaba por una definición biológica. Todos los textos de esta época tendrán ese sesgo orientado a las especulaciones científico-filosóficas.

10 "Mientras me era fácil amaestrar a un perro en distinguir un cuadrado de un circulo, no lograba ejercitarle en distinguir el azul del rojo; lo que en el laboratorio de Pavlov no se conseguía tampoco" (Nicolai, 1925a, p. 18). y maravillosamente adaptados a las necesidades del ambiente" (Nicolai, 1925a, p. 81).

La inteligencia, en su dependencia de la fisiología y su constante complejización filogenética, nace del instinto, pero se independiza de este. Es por ello que, en el inicio se presenta un tiempo y espacio ligados a las sensaciones y al instinto, pero luego, con la complejización de los reflejos, la inteligencia brinda una posibilidad de un tiempo que no está sujeta al tiempo instintivo, sensorial. Es el tiempo y el espacio de una "nueva metafísica" producida por la relatividad de Einstein. Las derivaciones filosóficas de los principios de la teoría de la relatividad que fueron publicadas en el libro se condensarían en Sentido filosófico de la teoría de la relatividad que publica en la célebre Revista de Filosofía de José Ingenieros, luego de la visita de Einstein a la Argentina en 1925 (Nicolai, 1925b). En ambos textos, Nicolai (1925a y 1925b) asevera que los métodos científicos han superado ampliamente a los filosóficos y critica los desarrollos de Ortega y Gasset, que por esas fechas tienen gran impacto en Argentina y Córdoba (Ferrari, 2012). Estas apreciaciones que enlazan la fisiología a la psicología, en una argumentación teórica terminan por concluir con una aseveración política. De hecho, en la misma línea de pensamiento sostenida por Pavlov, ambicionaba un programa eugenésico ${ }^{11}$.

\section{Entre la intelectualidad progresista y el conservadurismo cordobés}

En el caso de Nicolai, pudimos encontrar varias referencias de su relación con la intelectualidad progresista cordobesa. Esa afinidad con los intelectuales del movimiento de la Reforma Universitaria de 1918, puede explicar el hecho de que finalmente

11 Nicolai publicó en 1941 La eugenesia como gloriosa culminación de la medicina. Este, como otros textos, permite ubicar a Nicolai dentro de una trama de idearios políticos en donde la fisiología aparece como una ciencia aplicada al cambio social. A pesar de que sus reflejos condicionados indicaban el impacto que el medioambiente tenía en la conducta, Pavlov creía que el tipo ruso era un tipo poco perfeccionado y que un programa eugenésico alteraría el porvenir del destino de Rusia. Esta creencia probablemente se sustentaba en la influencia de su maestro de fisiología, Ilya F. Tsion (García, 2012). 
haya sido apartado de la cátedra de Fisiología. Había encontrado una ciudad que lo recibía amablemente tras el forzado exilio que sufrió. El Dr. Francisco De la Torre fue el principal gestor de la contratación de Nicolai, de orientación reformista y rector de la Universidad $^{12}$. En vísperas de la presentación del nuevo docente, fue De la Torre quién expresó las razones por las cuales se contrató a Nicolai:

Una agitación grande le ha sido necesaria para iniciarse en la liberación del yugo que el dogmatismo religioso o filosófico le había impuesto por tres siglos. (...) hemos buscado al hombre de ciencia positiva y lo presentamos hoy en la persona de los profesores doctores Nicolai y Goldschmidt. (De la Torre, 1922, p. 450)

Sin embargo, la intelectualidad reformista ya había incorporado la presencia de Nicolai desde hacía unos años, Deodoro Roca publicaba: "Hay que reconocer con Nicolai, que nunca, desde que los hombres hacen ciencia, se ha visto a los que son vanguardia en las luchas del espíritu, mostrarse tan entusiastas de la eficacia de la fuerza bruta (Roca, 1920, p. 378). Lo que indica que De la Torre fue, quizás, el instrumento ejecutor de un deseo forjado en el núcleo de intelectuales reformistas. Nicolai guardó gran simpatía por el movimiento y las ideas reformistas, que de hecho impulsó en la política universitaria. Sin embargo, como vimos, tenía poco en común con gran parte de los intelectuales cordobeses, afectados o por el vitalismo español de Ortega y Gasset o por el materialismo marxista.

Nicolai no parece haber impactado en la intelectualidad y la ciencia cordobesa de un modo decisivo. No hemos podido encontrar referencias concretas al posible impacto de las ideas del médico alemán o referencias a la obra de Pavlov. Esto es probable debido a que en la Facultad de Medicina tuvo una gran presencia una medicina ligada al pensamiento religioso. Por otro lado, una gran parte de la inte-

12 "Se autorizó al Sr. Rector para realizar los gastos que demande la venida del profesor Nicolai (...)” (p. 19) “(...13) Acta n.. 15. - Sesión ordinaria de los días 3 y 5 de agosto de 1921, celebrada bajo la presidencia del Sr. Rector, Dr. Francisco J. De la Torre en Actas y documentos oficiales (1922). lectualidad progresista cordobesa, que lo acogía y respetaba, no compartía su visión cientificista de la conducta humana. En Córdoba proliferó el vitalismo de Ortega y Gasset y Eugenio D'Ors, en contra de los cuales Nicolai manifestó pública aversión. Aun así, la desgraciada suerte del fisiólogo alemán le posibilitó una tolerancia benevolente por parte de intelectuales como Deodoro Roca. A menudo, esta lógica le permitió convivir, por un tiempo, con facciones ideológicas de compromisos opuestos. La particular razón por la que había sido expulsado de Alemania, su pacifismo, se tornó en moneda de cambio que valió tanto para progresistas como conservadores.

Apenas fue incorporado a la casa de estudios, fue elegido por los estudiantes para que formase parte del consejo directivo. En ese espacio es donde comenzó a gestarse la oposición que luego le llevaría a ser desplazado de su cargo. Luego de un año de haber ingresado, las tensiones entre reformistas y contrarreformistas crecían a cada instante. Hasta que el rector De la Torre solicitó la intervención de la Universidad. En el mismo momento el rector quedaría desplazado de su cargo y Antonio Sagarna comenzaría su labor de interventor para reorganizar la institución. Desde ese espacio político, el contrarreformismo tuvo su máxima expresión y Nicolai luchó en contra de lo más representativo del conservadurismo cordobés, el Dr. Brandán y el Dr. Morra, en particular respecto de la participación estudiantil en el Consejo ${ }^{13}$.

El resultado de estos desacuerdos políticos comenzaría a manifestarse paulatinamente; Nicolai

13 En las ordenanzas y resoluciones de carácter docente y administrativo dictadas por el H. Consejo directivo de la Facultad de Ciencias Médicas durante 1922, se discutió sobre la participación estudiantil, discusiones que quizás le costarían el cargo a Nicolai: Dr. Nicolai: Estoy conforme con la mayoría de la Comisión, (...) menos en lo que se refiere a la representación estudiantil. (...) tengo la plena seguridad de que en ninguna universidad de Europa los alumnos tienen menos derechos que aquí. (...)

Dr. Morra: (...) No, señor, decimos y sostenemos que no debe haber representación estudiantil (...)

Se vota el despacho (...). Por la afirmativa: Dres. Parga, Aliaga, Gómez, Morra, Brandán, Lazcano. Por la negativa: Arcidiácono, Lezama, Astraín, Strada, Nicolai. Resulta afirmativa. (Bases para la reforma de los estatutos universitarios. Sesión ordinaria del 14 de agosto de 1923 , pp. 10 -27). 
sería neutralizado por la cultura conservadora de la universidad cordobesa. Es por ello que la intelectualidad religiosa y conservadora no es una nota de color local, sino que es una particularidad cultural de gran injerencia. Nicolai explicará en las páginas de la Revista de Filosofía de Ingenieros que habiendo entablado una discusión sobre su contrato, no obtuvo respuestas sobre este punto hasta que finalmente:

El 9 de mayo se reunió el C. Directivo de la Facultad de Medicina, con asistencia de los doctores Lazcano, Sayago, Orrico, Martínez, Galíndez, Strada y Parga, excepción hecha de los consejeros estudiantiles Sayago y Orrico, el Consejo Directivo, (...), aprobó la siguiente resolución ignominiosa: declarar que el profesor Nicolai ha faltado al cumplimiento de su contrato (...) y poner en posesión de la cátedra al suplente doctor Barilari. (Editorial, Revista Filosofía, 1927, p. 390)

Estos gestos del Consejo Directivo movilizaron a la juventud estudiantil; más de 500 estudiantes se reunieron el 10 de mayo en el local de La Voz del Interior. Días después, en la noche del 21 de mayo de 1927, "La noche del 21 de mayo, en la Sociedad Unione e Benevolenza”. Allí quien pronunció unas palabras fue Deodoro Roca:

Y ese es, cabalmente, el que sabéis practicar. Desdén matizado por la dulce Ironía defendida por Anatole France: La que calma la cólera y nos enseña a mofarnos de los malvados y de los tontos, a quienes sin ella tendríamos la flaqueza de odiar. (Roca, en Editorial Revista de Filosofía, 1927, p. 392)

Al día siguiente, el 22, Nicolai daría una conferencia en un acto de desagravio, titulada La ciencia y la moral. Allí expondría con nombre y apellido los grupos del conservadurismo cordobés ${ }^{14}$ que habrían operado para apartarle de su cargo. Al abandonar

14 ¿Qué es lo que reúne a los Morra con los Martínez, a los Strada con los Lascano? ¿Cuál es la ideología común, en que pretendieron ampararse para justificar sus procederes? (Nicolai, 1927a, pp. 302-303). la cátedra escribe, Homenaje de despedida a la tradición de Córdoba docta y santa: "Se puede fiar en que en Córdoba no existe el peligro del modernismo" (Nicolai, 1927b, p. 57). En todo el desarrollo de este texto, prologado por Emilio Pizarro Crespo ${ }^{15}$, Nicolai se expresa con una ironía implacable y desnuda todas y cada una de las facetas de la vida universitaria cordobesa. No es trivial la fecha en que Nicolai es nuevamente separado de una cátedra universitaria: es el momento en que la Contrarreforma hace estragos en la Universidad y el movimiento de 1918 se ve diezmado por diferencias internas.

Luego de abandonar su cátedra en Córdoba, tal como lo menciona en una carta a Relgis, reconoce que ha estado viviendo como un vagabundo, pasando de Córdoba a la ciudad de Rosario para dictar, de 1927 a 1930, cursos de Sociología en la Universidad del Litoral. De 1931 a 1932, en Europa, da conferencias en la Unión Soviética y España, para, finalmente, volver a Argentina. En Buenos Aires, dicta cursos en el Colegio Libre de Estudios Superiores (CLES), un espacio de formación instrumentado por la intelectualidad progresista, y finaliza en 1931 en Santiago de Chile, en la cátedra de Fisiología en la Facultad de Veterinaria. En esa misma ciudad, muere en 1964 a los noventa años.

Nicolai mantuvo vínculos con la intelectualidad de la izquierda argentina. Sus textos fueron publicados por las editoriales Claridad ${ }^{16}$, Imán y por el Colegio Libre de Estudios Superiores ${ }^{17}$ que edita en español su texto Biología de la guerra, en 1932. Este texto fue prologado por el conocido premio Nobel,

15 Médico relacionado con el grupo de intelectuales progresistas de la Reforma Universitaria. Mantuvo lazos con Deodoro Roca, Jorge Orgaz, Gregorio Bermann, entre otros. Inicialmente, tuvo una gran cercanía con el marxismo. En 1935 fue invitado, por la sociedad soviética de contactos culturales internacionales (VOKS), a visitar los servicios de salud en Rusia. Luego participaría, junto a Gregorio Bermann, en la Guerra Civil española.

16 Fue fundada por Antonio Zamora, estaba ubicada en la calle Boedo 837, comenzando a funcionar el 30 de enero de 1922. Su importancia fue fundamental ya que ahí se formó el "Grupo Boedo", integrado por escritores como Elías Castelnuovo, Álvaro Yunque, Leónidas Barletta, Nicolás Olivari, Roberto Marini, César Tiempo, Raúl González Tuñón y artistas plásticos como Agustín Riganelli, Guillermo Facio Hebecquer y Lorenzo Stanchina.

17 El Colegio Libre de Estudios Superiores fue un espacio de amplitud intelectual que tenía como principal objetivo la difusión del antifascismo. 
Romain Rolland, quien junto a Manuel Gálvez, le escriben un pequeño libro titulado Nicolai, el gran europeo (Rolland, s. f.). En los últimos momentos de Nicolai en Córdoba, fue despedido por Emilio Pizarro Crespo, que escribe en el homenaje de despedida: "Al Doctor Jorge Nicolai: (...) la Catedral, confuso monumento, y la Universidad, que es un asilo. (...) i¿Cuándo saldrá, Doctor, de este agujero?!". Líneas que abren, a modo de prólogo, el texto con el que Nicolai se despide de Córdoba. Del mismo modo, Deodoro Roca escribió en 1932 una conferencia en honor a una visita de Nicolai, en la que declara:

Jorge Federico Nicolai, especie de Sócrates vagabundo, que ha sobrevivido a la cicuta, (...) encarcelado durante meses por el delito de pensar que la guerra es mala y que el régimen de la propiedad privada debe cambiarse, exiliado luego, erguido siempre. (Roca, 1932 en Sanguinetti, 2003, pp. 108-109)

Estas palabras de Deodoro, un vitalista D'Orsiano convencido, defensor de las ideas de Ortega y Gasset, nos ilustran muy bien sobre el tipo de acogida que Nicolai tuvo en la intelectualidad progresista. Como hemos mencionado, esta pudo ser una de las razones por las cuales las ideas de Nicolai no tuvieron mayor difusión e impacto. Es decir, el grupo de intelectuales reformistas acogió al médico alemán no por un interés en adoptar sus teorías, sino por el respeto a la estatura intelectual y la simpatía con los ideales pacifistas. No solo esto, sino que además de su contacto con el grupo reformista cordobés, estableció una relación con Elías Castelnuovo, quien fuera referente del grupo de Boedo ${ }^{18} \mathrm{y}$ fundador de la revista Extrema izquierda. Esta vinculación se hará evidente, pues Nicolai publicó, como mencionamos, apoyado por la editorial Claridad. Está claro que Nicolai distaba

18 El grupo Boedo fue un agrupamiento de artistas de vanguardia de la Argentina durante la década de 1920. Tradicionalmente, la historiografía cultural argentina lo opuso al grupo Florida. Recibieron ese nombre porque uno de sus puntos de confluencia era la editorial Claridad. El grupo se caracterizó por su temática social, sus ideas de izquierda y su deseo de vincularse con los sectores populares. de pertenecer a la izquierda, pero simpatizaba con algunos principios del Reformismo Universitario, no obstante podría decirse que comulgaba con una postura conservadora. Por otra parte, la intelectualidad de izquierda cordobesa, comprometida con el materialismo y las ideas de Marx, como es el caso de Gregorio Bermann, si bien pueden haber encontrado simpatías con el médico alemán, probablemente no compartiesen las críticas que Nicolai desplegó en torno del marxismo:

(...) Marx era un dialéctico, esto es, un iluso idealista. Venéresele cuanto se quiera, y como merece, pues ha hecho realmente muchísimo para la humanidad y también para la ciencia; pero el hecho está en que es imposible en cuanto es dialéctico, sea al mismo tiempo científico; son dos nociones tan diametralmente opuestas como el agua y el fuego. (Nicolai 1936, p. 17)

De hecho, también cuestionó la postulación de la lucha de clases. Sin embargo, además de las deferencias que los intelectuales de orientación vitalista y marxista pudieron tener con el fisiólogo alemán, dentro del ámbito de izquierda también se lo convocó como agente neutro e instruido. En el libro La Rusia actual y futura (1932), una serie de conferencias compiladas, Sergio Bagú convocaba a Nicolai como crítico neutral:

En cuanto a la Nueva Rusia, crisol fecundo donde se gesta una humanidad mejor, pensamos que la hiere tanto el panegírico incondicional como la agresión grotesca de las piaras. (Bagú en Nicolai, 1932, p. 6)

Debo advertir que mi exposición ha de ser el fruto de un estudio objetivo y desapasionado. (Nicolai, 1932, p. 7)

\section{Discusión}

Tal como pudimos observar, el impacto de la obra de Iván Pavlov en la obra de Nicolai varió en virtud de las posibilidades técnicas de su lugar de residencia. El primer período estuvo marcado por el aprendizaje del método y por la apreciación simbólica de la 
posibilidad de su utilización en estudios psicológicos objetivos. En el período en que estuvo en la ciudad de Córdoba, probablemente por carecer de medios técnicos, se nota una preponderancia de la valoración de los aspectos teóricos de las teorías de Pavlov.

Se podría realizar una triple estratificación en la postura que expuso Nicolai en su obra editada en su estancia en Córdoba, donde Darwin brindaría los fundamentos de la conducta animal y humana, y una perspectiva evolutiva a partir de la cual Spencer lograría una interpretación de los complejos fenómenos sociales, Pavlov mostraría los mecanismos fisiológicos de los fenómenos psicológicos y Einstein brindaría un marco general en el que la física sería el marco de una nueva "metafísica" científica que abarcaría a los demás campos del saber humano.

Esta triple estratificación, que lo aleja de la psicología de los pueblos, de la psicología experimental alemana y de la psicología experimental de tinte clínico francés, probablemente haya producido que sus tesis no hayan sido aceptadas y difundidas en el marco de un medio cultural que no encontraba interlocutores interesados. Esta postura se entrelaza con una perspectiva política un tanto conservadora, pacifista, distante y crítica del pensamiento de izquierda. Sin embargo, su involucramiento con facciones progresistas y hasta de izquierda estuvo posibilitado por la veneración a la estatura intelectual del sabio alemán y una bienvenida que buscó resguardarlo de las injusticias de las épocas de la guerra y las políticas ultraconservadoras. Su presencia también fue ampliamente tolerada por su posición de intelectual que brinda un saber neutral amparado por el talante de la ciencia.

\section{Referencias}

Actos y documentos oficiales. (1922) Consejo Superior. Sesión ordinaria de los días 3 y 5 de agosto de 1921. Revista de la Universidad Nacional de Córdoba, 11, año 9, n.. 8,9 y 10, 1-104.

Bases para la reforma de los estatutos universitarios. Sesión ordinaria del 14 de agosto de 1923. (1923). Revista de la Universidad Nacional de Córdoba, vol. II, año 10, 10-48.
Bandrés, J., \& Llavona, R. (2003). Pavlov in Spain. The Spanish Journal of Psychology, 6(2), 81-92. Recuperado de http://www.ncbi.nlm.nih.gov/ pubmed/14628695

Cabello, F. (2013). El gran europeo Georg Friedrich Nicolai: médico y pacifista. Revista Médica de Chile, 141, 535-539.

Un grave conflicto universitario. Jorge F. Nicolai y la reacción conservadora en Córdoba. (1927). [Editoriall. Revista de Filosofía, 13, 386-395.

Danziger, K. (1983). Origins and basic principles of Wundt's Völkerpsychologie. British Journal of Social Psychology, 22(4), 303-313.

Danziger, K. (2010). Problematic encounter: Talks on psychology and history. Disponible en http://www. kurtdanziger.com/Problematic\%20Encounter3.pdf

De la Torre, F. (1922). Recepción de los profesores Nicolai y Goldschmidt. Revista de Filosofía, 9(1), 59-76.

Ferrari, F. J. (2012). Entre el dispositivo psiquiátrico y la disciplina monacal : una historia genealógica de las primeras lecturas de la psicopatología freudiana en Córdoba (1758-1930). (Tesis de doctorado inédita). Universidad Nacional de Córdoba, Argentina.

Ferrari, F. J. (2013). Virgilio Ducceschi y el primer laboratorio de psicología experimental en Córdoba, Argentina (1907). Estudos E Pesquisas Em Psicologia, 13(2). Disponible en http://www.revispsi.uerj. br/v13n2/artigos/html/ v13n2a21.html

García, L. N. (2012). La recepción de la psicología soviética en la Argentina: lecturas y apropiaciones en psicología, psiquiatría y psicoanálisis (1936-1991). (Tesis de doctorado en Psicología). Universidad de Buenos Aires, Argentina.

Goodwin, C. J. (1991). Misportraying Pavlov's apparatus. The American Journal of Psychology, 104(1), 135-141.

Goodwin, C. J. (2009). Historia de la psicología moderna. México. Limusa Wiley.

Grundmann, S. (2004). The Einstein dossiers. Science and politics - Einstein's Berlin period. Berlin: Springer.

Heilbron, J. M., Guilhot, N., \& Laurent, J. (2008). Toward a transnational history of the social sciences. Journal of the History of the Behavioral Sciences, 44(2), 146-160. 
Kästner, I. (2005). Der Deutsche Arzt und Pazifist Georg Friedrich Nicolai (1874-1964) als Schüler des russischen Physiologen Ivan Petrovic Pavlov (1849-1936). [El médico y pacifista alemán Georg Friedrich Nicolai (1874-1964) como pupilo del fisiólogo ruso Ivan Petrovic Pavlov (1849-1936)] Würzburger Medizinhistor, 24, 261-267.

Klappenbach, H. (1994). La recepción de Wundt en la Argentina. 1907: creación del Segundo Curso de Psicología en la Universidad de Buenos Aires. Revista de Historia de la Psicología, 15(1-2), 181-197.

Nicolai, G. F. (1908). Das Lernen der tiere (auf Grund von Versuchen mit Pawlowscher Seichelfistel). Separatabdruck aus Zentralblatt für Physiologie, Bd. XXII. Vergandlungen der physiologischen Gesellschaft zu Berlin, 11.

Nicolai, G. F. (1918). The biology of war (C. A. Grande \& J. Grande, Trads.). New York: The Century Co. Recuperado de http://www.archive.org/details/ biologyofwarOOnicouoft

Nicolai, G. F. (1922). La vida. Revista de Filosofía, 8(46), $65-76$.

Nicolai, G. F. (1923). Las isopsicas de los animales y la relación entre la inteligencia y el cerebro según observaciones en 192 perros. Revista de la Universidad Nacional de Córdoba, 10(7-8), 3-44.

Nicolai, G. F. (1925a). La base biológica del relativismo científico y sus complementos absolutos. Revista de la Universidad Nacional de Córdoba, 12(1-3), 1-182.

Nicolai, G. F. (1925b). Sentido filosófico de la teoría de la relatividad. Revista de Filosofía, 22, 1-26.

Nicolai, G. F. (1926). In memoriam ingenieros. Revista de Filosofía, 1, 83-90.

Nicolai, G. F. (1927a). La ciencia y la moral. Revista de Filosofía, 13(3), 301-317.

Nicolai, G. F. (1927b). Homenaje de la despedida a la tradición de Córdoba docta y santa. Buenos Aires: Sociedad de Publicaciones El Inca.

Nicolai, G. F. (1930). La influencia de los estudios puros en la formación de la nueva conciencia. Universidad Nacional de La Plata, Extensión Universitaria

Nicolai, G. F. (1932). La Rusia actual y futura. Ciencias politicas (Conferencias, n.․ㅜ 7). Buenos Aires: Editorial Claridad.
Nicolai, G. F. (1936). Fundamentos reales de la sociología. Santiago de Chile: Biblioteca Ercilla.

Nicolai, G. F. (1947). La eugenesia como gloriosa culminación de la medicina. Buenos Aires: Editorial SAC.

Ramón, L. (1997). Rumbo al nuevo mundo: cuatro psicólogos de Europa Oriental en la historia de la psicología en América del Sur. Revista Latinoamericana de Psicología, 29(1), 9-34.

Roca, D. (1920). La Universidad y el espíritu libre. Revista de la Universidad Nacional de Córdoba, 3(5-6), 377-383.

Rolland, R. (s. f.). Nicolai el gran europeo. Buenos Aires: Pax.

Rowe, D. E., \& Schulmann, R. (2007). Einstein on politics. Princeton: Princeton University Press. Recuperado de http://press.princeton.edu/chapters/s8359.html

Ruíz, G., Sánchez, N., \& De la Casa, L. G. (2003). Pavlov in America: A heterodox approach to the study of his influence. The Spanish Journal of Psychology, 6(2), 99-111. Recuperado de http://www.redalyc. org/articulo.oa?id=17260204

Sanguinetti, H. (2003). Trayectoria de una flecha. Las obras y los días de Deodoro Roca. Buenos Aires: Librería Histórica.

Todes, D. P. (2003). From lone investigator to laboratory chief: Ivan Pavlov's research notebooks as a reflection of his managerial and interpretive style. En F. L. Holmes, J. Renn \& H. J. Rheinberger (Eds.), Reworking the bench: Research notebooks in the history of science (pp. 203-220). Amsterdam, Netherlands: Kluwer Academic Publishers.

Watson, J. B. (1913). Psychology as the behaviorist views it. Psychological Review, 20, 158-277.

Watson, J. B. (1914). Behavior: An introduction to comparative psychology. New York: Holt.

Windholz, G. (1995). Pavlov on the conditioned reflex method and its limitations. The American Journal of Psychology, 108(4), 575-588. Recuperado de http://www.jstor.org/stable/1423074

Zuelzer, W. (1982). The Nicolai case. Detroit, MI: Wayne State University Press. 